ALEA, Lat. Am. J. Probab. Math. Stat. 16, 809-816 (2019)

DOI: 10.30757/ALEA.v16-29

\title{
Order of fluctuations of the free energy in the SK model at critical temperature
}

\section{Wei-Kuo Chen and Wai-Kit Lam}

School of Mathematics, University of Minnesota

206 Church Street SE,

Minneapolis, MN 55455, USA.

E-mail address: \{ wkchen, wlam \} @umn.edu

Abstract. We present an elementary approach to the order of fluctuations for the free energy in the Sherrington-Kirkpatrick mean field spin glass model at and near the critical temperature. It is proved that at the critical temperature the variance of the free energy is of $O\left((\log N)^{2}\right)$. In addition, we show that if one approaches the critical temperature from the low temperature regime at the rate $O\left(N^{-\alpha}\right)$ for some $\alpha>0$, then the variance is of $O\left((\log N)^{2}+N^{1-\alpha}\right)$.

\section{Introduction}

The Sherrington-Kirkpatrick (SK) model was initially introduced in Sherrington and Kirkpatrick (1975) in order to explain some strange magnetic properties of certain alloys. Over the past decades, it has received a great attention in the physics and mathematics communities. See Mézard et al. (1987) for physics treatments and Panchenko (2013); Talagrand (2003, 2011a,b) for recent mathematical progress.

The aim of this short note is to study the order of fluctuations of the free energy in the SK model at the critical temperature. For any $N \geq 1$, the Hamiltonian of the SK model is defined as

$$
H_{N}(\sigma)=\frac{1}{\sqrt{N}} \sum_{1 \leq i, j \leq N} g_{i j} \sigma_{i} \sigma_{j}
$$

for any $\sigma \in \Sigma_{N}:=\{-1,+1\}^{N}$, where $\left(g_{i j}\right)_{1 \leq i, j \leq N}$ is a family of independent standard Gaussian random variables. The covariance of $H_{N}$ is described by

$$
\mathbb{E} H_{N}\left(\sigma^{1}\right) H_{N}\left(\sigma^{2}\right)=N R\left(\sigma^{1}, \sigma^{2}\right)^{2},
$$

where $R\left(\sigma^{1}, \sigma^{2}\right):=N^{-1} \sum_{i=1}^{N} \sigma_{i}^{1} \sigma_{i}^{2}$ is called the overlap between spin configurations $\sigma^{1}, \sigma^{2} \in \Sigma_{N}$. The free energy and the Gibbs measure at (inverse) temperature

Received by the editors February 25th, 2019; accepted May 4th, 2019.

2010 Mathematics Subject Classification. 60K35, 82B44.

Key words and phrases. Spin glass, Sherrington-Kirkpatrick model, Disorder chaos. 
$\beta>0$ is defined as

$$
F_{N}(\beta)=\log \sum_{\sigma \in \Sigma_{N}} \exp \left(\beta H_{N}(\sigma)\right)
$$

and

$$
G_{N}(\sigma)=\frac{\exp \left(\beta H_{N}(\sigma)\right)}{Z_{N}(\beta)}, \forall \sigma \in \Sigma_{N}
$$

where $Z_{N}(\beta):=\sum_{\sigma \in \Sigma_{N}} \exp \left(\beta H_{N}(\sigma)\right)$. Denote by $\sigma^{1}, \sigma^{2}$ i.i.d. samplings from $G_{N}$ and by $\langle\cdot\rangle$ the expectation with respect to these random variables.

The SK model is known to exhibit a phase transition at the critical temperature $\beta_{c}:=1 / \sqrt{2}$. In the high temperature regime $\beta<\beta_{c}$, the limiting free energy is equal to the anneal free energy, that is,

$$
\lim _{N \rightarrow \infty} \frac{F_{N}(\beta)}{N}=\lim _{N \rightarrow \infty} \frac{\log \mathbb{E} Z_{N}}{N}
$$

and if we sample two independent $\sigma^{1}, \sigma^{2}$ from the Gibbs measure, then they are essentially orthogonal to each other in the sense that $\lim _{N \rightarrow \infty} \mathbb{E}\left\langle R\left(\sigma^{1}, \sigma^{2}\right)^{2}\right\rangle=0$. In contrast, the model exhibits different behaviors in the lower temperature regime $\beta>\beta_{c}$, where we see that

$$
\lim _{N \rightarrow \infty} \frac{F_{N}(\beta)}{N}<\lim _{N \rightarrow \infty} \frac{\log \mathbb{E} Z_{N}}{N}
$$

and the two independent samplings $\sigma^{1}, \sigma^{2}$ have nonzero overlap, that is,

$$
\lim _{N \rightarrow \infty} \mathbb{E}\left\langle R\left(\sigma^{1}, \sigma^{2}\right)^{2}\right\rangle>0
$$

Indeed, it was conjectured that the limiting distribution of $R\left(\sigma^{1}, \sigma^{2}\right)$ should be be described by a probability measure whose support is an interval $[0, q]$ for some $q \in(0,1)$. See Mézard et al. (1987); Talagrand (2003, 2011b) for more details.

Previous rigorous results on the order of fluctuations of the free energy is summarized as follows:

- High temperature: Aizenman, Lebowitz, and Ruelle (Aizenman et al., 1987) proved that the free energy has Gaussian fluctuations in the high temperature regime. Their result implies that the limit of $\operatorname{Var}\left(F_{N}(\beta)\right)$ exists and is finite.

- Near critical temperature: The problem of understanding the transition near the critical temperature was intensively studied in Talagrand's books Talagrand (2003, Section 2.14) and Talagrand (2011b, Section 11.7), where he showed that when $\beta=\beta_{N}$ approaches the critical temperature from the high temperature regime in the rate $\lim _{N \rightarrow \infty} N^{1 / 3}\left(\beta_{c}^{2}-\beta_{N}^{2}\right)=c$, then the overlap undergoes a phase transition depending on whether $c$ is finite or infinite. Exactly at the criticality $\beta_{c}$, he also proved that the overlap is controlled by $\mathbb{E}\left\langle R\left(\sigma^{1}, \sigma^{2}\right)^{2}\right\rangle \leq C / \sqrt{N}$ for some universal constant $C$, from which it can be derived that $\operatorname{Var}\left(F_{N}\left(\beta_{c}\right)\right) \leq C \sqrt{N}$, see Remark 2.2 below.

- Low temperature: Chatterjee (2009) showed that

$$
\operatorname{Var}\left(F_{N}(\beta)\right) \leq \frac{C(\beta) N}{\log N}
$$


where $C(\beta)$ is a constant independent of $N$. Incidentally, it was proved by Chatterjee $(2019+$, Theorem 2.4) that fluctuations of the free energy are at least of order 1 at any $\beta$.

The order of fluctuations of the free energy is also studied in the physics literature, for example Aspelmeier (2008); Aspelmeier et al. (2008); Parisi and Rizzo (2009). At the critical temperature, it is expected that

$$
\operatorname{Var}\left(F_{N}\left(\beta_{c}\right)\right)=\frac{1}{6} \log N+O(1) .
$$

In the low temperature regime, it is believed that $\operatorname{Var}\left(F_{N}(\beta)\right) \asymp N^{2 \mu}$ for some $\mu \geq 0^{1}$. However, physicists' simulations have not reached an agreement: while some suggested $\mu=1 / 4$, it was also proposed that $\mu=1 / 6$. See Aspelmeier et al. (2008, Section 7) for a detailed discussion. Notably, Aspelmeier (2008) argued that $\mu \leq 1 / 4$.

This note is focused on the order of fluctuation of $F_{N}$ at the critical temperature. Our main result below contains two parts. First, at the critical temperature, we obtain an upper bound of order $(\log N)^{2}$ for the variance of the free energy. Second, we show that if one approaches the critical temperature from the low temperature regime in the rate $\beta_{N}=\sqrt{\beta_{c}^{2}+N^{-\alpha}}$ for $\alpha \in(0,1)$, then a polynomial bound $N^{1-\alpha}$ is obtained. This improves Chatterjee's bound $N / \log N$ in the near critical case.

Theorem 1.1. The following statements hold:

(1) There exists a constant $C>0$ such that

$$
\operatorname{Var}\left(F_{N}\left(\beta_{c}\right)\right) \leq C\left((\log N)^{2}+1\right), \forall N \geq 1 .
$$

(2) For any fixed $\alpha>0$ and $d>0$, there exists a constant $C$ depending only on $\alpha$ and $d$ such that

$$
\operatorname{Var}\left(F_{N}\left(\sqrt{\beta_{c}^{2}+d N^{-\alpha}}\right)\right) \leq C\left((\log N)^{2}+N^{1-\alpha}\right), \forall N \geq 1 .
$$

Our approach is motivated by a work of Guerra and Toninelli (Guerra and Toninelli, 2002), where they derived the limit of the SK free energy and provided a rate of convergence. Their idea was to consider a coupled free energy with Hamiltonian of the form $\sqrt{s}\left(H_{N}\left(\sigma^{1}\right)+H_{N}\left(\sigma^{2}\right)\right)+\lambda N R\left(\sigma^{1}, \sigma^{2}\right)^{2}$, where $\sqrt{s}$ is a varying temperature and $\lambda$ is an auxiliary parameter. From this, they derived an ordinary differential inequality for this coupled free energy in the variable $s \geq 0$ and by solving this inequality, they obtained the rate of convergence of the free energy. Our argument adopts a different route by considering a coupled Hamiltonian that is related to the problem of chaos in disorder in the SK model considered in Chatterjee (2009, 2014).

\section{Proof of Theorem 1.1}

Let $H_{N}^{\prime}, H_{N}^{\prime \prime}$ be two independent copies of $H_{N}$. For $0 \leq t \leq 1$ and $\sigma, \rho \in \Sigma_{N}$, set

$$
\begin{aligned}
H_{N, t}^{1}(\sigma) & =\sqrt{t} H_{N}(\sigma)+\sqrt{1-t} H_{N}^{\prime}(\sigma), \\
H_{N, t}^{2}(\rho) & =\sqrt{t} H_{N}(\rho)+\sqrt{1-t} H_{N}^{\prime \prime}(\rho) .
\end{aligned}
$$

Note that

$$
\mathbb{E} H_{N, t}^{1}(\sigma) H_{N, t}^{2}(\rho)=t N R(\sigma, \rho)^{2} .
$$

\footnotetext{
${ }^{1} a_{N} \asymp b_{N}$ means that there exist $c, C>0$ independent of $N$ such that $c b_{N} \leq a_{N} \leq C b_{N}$.
} 
For any $t \in[0,1]$ and $\lambda \in \mathbb{R}$, define

$$
\phi_{N}(t, \lambda)=\frac{1}{N} \mathbb{E} \log \sum_{\sigma, \rho \in \Sigma_{N}} \exp \left(\beta\left(H_{N, t}^{1}(\sigma)+H_{N, t}^{2}(\rho)\right)+\lambda \beta^{2} N R(\sigma, \rho)^{2}\right) .
$$

Denote by $\langle\cdot\rangle_{t, \lambda}$ the Gibbs expectation associated to this free energy. That is, the expectation with respect to the measure

$$
\frac{\sum_{\sigma, \rho} I((\sigma, \rho) \in \cdot) \exp \left(\beta\left(H_{N, t}^{1}(\sigma)+H_{N, t}^{2}(\rho)\right)+\lambda \beta^{2} N R(\sigma, \rho)^{2}\right)}{\sum_{\sigma, \rho} \exp \left(\beta\left(H_{N, t}^{1}(\sigma)+H_{N, t}^{2}(\rho)\right)+\lambda \beta^{2} N R(\sigma, \rho)^{2}\right)} .
$$

Lemma 2.1. For any $\beta>0$ and $t \in[0,1]$ satisfying $2 \beta^{2} t<1$, we have that for any $N \geq 1$,

$$
\mathbb{E}\left\langle R(\sigma, \rho)^{2}\right\rangle_{t, 0} \leq \frac{2}{N\left(1-2 \beta^{2} t\right)} \log \frac{2}{1-2 \beta^{2} t} .
$$

Proof: Note that for $t \in(0,1)$ and any $\sigma, \sigma^{\prime}, \rho, \rho^{\prime} \in \Sigma_{N}$,

$$
\begin{aligned}
& \mathbb{E}\left(\frac{H_{N}(\sigma)}{\sqrt{t}}-\frac{H_{N}^{\prime}(\sigma)}{\sqrt{1-t}}\right) H_{N, t}^{1}\left(\sigma^{\prime}\right)=0, \\
& \mathbb{E}\left(\frac{H_{N}(\rho)}{\sqrt{t}}-\frac{H_{N}^{\prime \prime}(\rho)}{\sqrt{1-t}}\right) H_{N, t}^{2}\left(\rho^{\prime}\right)=0, \\
& \mathbb{E}\left(\frac{H_{N}(\sigma)}{\sqrt{t}}-\frac{H_{N}^{\prime}(\sigma)}{\sqrt{1-t}}\right) H_{N, t}^{2}\left(\rho^{\prime}\right)=N R\left(\sigma, \rho^{\prime}\right)^{2}, \\
& \mathbb{E}\left(\frac{H_{N}(\rho)}{\sqrt{t}}-\frac{H_{N}^{\prime}(\rho)}{\sqrt{1-t}}\right) H_{N, t}^{1}\left(\sigma^{\prime}\right)=N R\left(\sigma^{\prime}, \rho\right)^{2} .
\end{aligned}
$$

Using Gaussian integration by parts gives

$$
\begin{aligned}
\partial_{t} \phi_{N}(t, \lambda) & =\frac{1}{2 N} \beta \mathbb{E}\left\langle\left(\frac{H_{N}(\sigma)}{\sqrt{t}}-\frac{H_{N}^{\prime}(\sigma)}{\sqrt{1-t}}\right)+\left(\frac{H_{N}(\rho)}{\sqrt{t}}-\frac{H_{N}^{\prime \prime}(\rho)}{\sqrt{1-t}}\right)\right\rangle_{t, \lambda} \\
& =\frac{\beta^{2}}{2}\left(2 \mathbb{E}\left\langle R(\sigma, \rho)^{2}\right\rangle_{t, \lambda}-\mathbb{E}\left\langle R\left(\sigma^{1}, \rho^{2}\right)^{2}\right\rangle_{t, \lambda}-\mathbb{E}\left\langle R\left(\sigma^{2}, \rho^{1}\right)^{2}\right\rangle_{t, \lambda}\right) \\
& =\beta^{2}\left(\mathbb{E}\left\langle R(\sigma, \rho)^{2}\right\rangle_{t, \lambda}-\mathbb{E}\left\langle R\left(\sigma^{1}, \rho^{2}\right)^{2}\right\rangle_{t, \lambda}\right),
\end{aligned}
$$

where the pairs $(\sigma, \rho),\left(\sigma^{1}, \rho^{1}\right)$, and $\left(\sigma^{2}, \rho^{2}\right)$ are i.i.d. copies from the Gibbs measure $\langle\cdot\rangle_{t, \lambda}$ and the second equality used symmetry between the distributions corresponding to the pairs $\left(\sigma^{1}, \rho^{2}\right)$ and $\left(\sigma^{2}, \rho^{1}\right)$. Set

$$
\Phi_{N}(t, \lambda)=\phi_{N}(t, \lambda-t) .
$$

Then from the above equation,

$$
\begin{aligned}
\partial_{t} \Phi_{N}(t, \lambda) & =\partial_{t} \phi_{N}(t, \lambda-t)-\partial_{\lambda} \phi_{N}(t, \lambda-t) \\
& =\beta^{2}\left(\mathbb{E}\left\langle R(\sigma, \rho)^{2}\right\rangle_{t, \lambda-t}-\mathbb{E}\left\langle R\left(\sigma^{1}, \rho^{2}\right)^{2}\right\rangle_{t, \lambda-t}\right)-\beta^{2} \mathbb{E}\left\langle R(\sigma, \rho)^{2}\right\rangle_{t, \lambda-t} \\
& =-\beta^{2} \mathbb{E}\left\langle R\left(\sigma^{1}, \rho^{2}\right)^{2}\right\rangle_{t, \lambda-t} .
\end{aligned}
$$


Note that $\partial_{t} \Phi_{N}(s, \lambda+t)=-\beta^{2} \mathbb{E}\left\langle R\left(\sigma^{1}, \rho^{2}\right)^{2}\right\rangle_{s, \lambda+t-s}$. It follows that

$$
\begin{aligned}
\phi_{N}(t, \lambda) & =\Phi_{N}(t, \lambda+t) \\
& =\int_{0}^{t} \partial_{t} \Phi_{N}(s, \lambda+t) d s+\Phi_{N}(0, \lambda+t) \\
& \leq \Phi_{N}(0, \lambda+t) \\
& =\phi_{N}(0, \lambda+t) .
\end{aligned}
$$

Now using the convexity of $\phi_{N}$ in $\lambda$ gives

$$
\begin{aligned}
\beta^{2} \lambda \mathbb{E}\left\langle R(\sigma, \rho)^{2}\right\rangle_{t, 0} & =\lambda \partial_{\lambda} \phi_{N}(t, 0) \\
& \leq \phi_{N}(t, \lambda)-\phi_{N}(t, 0) \\
& \leq \phi_{N}(0, \lambda+t)-\phi_{N}(t, 0) \\
& =\phi_{N}(0, \lambda+t)-\phi_{N}(0,0),
\end{aligned}
$$

where the last equation used that $\phi_{N}(t, 0)=\phi_{N}(0,0)$. Note that under the measure $\mathbb{E}\langle\cdot\rangle_{0,0}, \sigma$ and $\rho$ are independent uniform random variables on $\Sigma_{N}$ and hence, $N R(\sigma, \rho)$ is equal to the sum of $N$ i.i.d. Rademacher random variables $X_{1}, \ldots, X_{N}$ in distribution. It is well-known (see, e.g., Talagrand, 2011b, Eq. (A.19)) that

$$
\mathbb{E} \exp \left[x\left(\frac{X_{1}+\cdots+X_{N}}{\sqrt{N}}\right)^{2}\right] \leq \frac{1}{\sqrt{1-2 x}}, \forall x \in[0,1 / 2)
$$

Consequently, using (2.1) and Jensen's inequality, we have

$$
\begin{aligned}
\beta^{2} \lambda \mathbb{E}\left\langle R(\sigma, \rho)^{2}\right\rangle_{t, 0} & \leq \phi_{N}(0, \lambda+t)-\phi_{N}(0,0) \\
& =\frac{1}{N} \mathbb{E} \log \left\langle\exp \left(\beta^{2}(\lambda+t) N R(\sigma, \rho)^{2}\right)\right\rangle_{0,0} \\
& \leq \frac{1}{N} \log \mathbb{E}\left\langle\exp \left(\beta^{2}(\lambda+t) N R(\sigma, \rho)^{2}\right)\right\rangle_{0,0} \\
& \leq \frac{1}{N} \log \frac{1}{\sqrt{1-2 \beta^{2}(\lambda+t)}}
\end{aligned}
$$

whenever $2 \beta^{2}(\lambda+t)<1$. In particular, plugging

$$
\lambda=\frac{1}{2}\left(\frac{1}{2 \beta^{2}}-t\right)
$$

into the above inequality completes our proof.

Proof of Theorem 1.1: Recall from Chatterjee (2009) that the variance of the free energy can be expressed as

$$
\operatorname{Var}\left(F_{N}(\beta)\right)=\beta^{2} N \int_{0}^{1} \mathbb{E}\left\langle R(\sigma, \rho)^{2}\right\rangle_{t, 0} d t
$$


For any $0<\delta<1 /\left(2 \beta^{2}\right) \leq 1$,

$$
\begin{aligned}
\int_{0}^{\frac{1}{2 \beta^{2}}-\delta} \mathbb{E}\left\langle R(\sigma, \rho)^{2}\right\rangle_{t, 0} d t & \leq \frac{1}{N} \int_{0}^{\frac{1}{2 \beta^{2}}-\delta} \frac{2}{1-2 \beta^{2} t} \log \frac{2}{1-2 \beta^{2} t} d t \\
& =\left.\frac{1}{2 N \beta^{2}}\left(\log \frac{1-2 \beta^{2} t}{2}\right)^{2}\right|_{0} ^{\frac{1}{2 \beta^{2}}-\delta} \\
& =\frac{1}{2 N \beta^{2}}\left(\left(\log \left(\beta^{2} \delta\right)\right)^{2}-(\log 2)^{2}\right) \\
& \leq \frac{1}{2 N \beta^{2}}\left(\log \left(\beta^{2} \delta\right)\right)^{2} \\
& \leq \frac{1}{N \beta^{2}}\left((\log \delta)^{2}+4(\log \beta)^{2}\right)
\end{aligned}
$$

where we have used the inequality $(\log (a b))^{2} \leq 2(\log a)^{2}+2(\log b)^{2}$ for any $a, b>0$. On the other hand, noting that $|R(\sigma, \rho)| \leq 1$ implies

$$
\int_{\frac{1}{2 \beta^{2}}-\delta}^{1} \mathbb{E}\left\langle R(\sigma, \rho)^{2}\right\rangle_{t, 0} d t \leq 1-\frac{1}{2 \beta^{2}}+\delta .
$$

From this,

$$
\operatorname{Var}\left(F_{N}(\beta)\right) \leq \beta^{2}\left(\frac{1}{\beta^{2}}\left((\log \delta)^{2}+4(\log \beta)^{2}\right)+\left(1-\frac{1}{2 \beta^{2}}+\delta\right) N\right) .
$$

If $\beta=\beta_{c}$, we take $\delta=1 / N$ so that

$$
\operatorname{Var}\left(F_{N}(\beta)\right) \leq\left((\log N)^{2}+4(\log 2)^{2}\right)+\frac{1}{2}
$$

and this gives the first assertion. If $\beta^{2}=\beta_{c}^{2}+d N^{-\alpha}$ for $d>0$, we take $\delta=d N^{-\alpha}$ and note that

$$
1-\frac{1}{2 \beta^{2}}+\delta=\frac{2 d N^{-\alpha}}{1+2 d N^{-\alpha}}+d N^{-\alpha} \leq 3 d N^{-\alpha},
$$

which implies that as long as $N$ is large enough,

$$
\begin{aligned}
\operatorname{Var}\left(F_{N}(\beta)\right) & \leq \beta^{2}\left(\frac{1}{\beta^{2}}\left((-\alpha \log N+\log d)^{2}+4(\log \beta)^{2}\right)+3 d N^{1-\alpha}\right) \\
& =(-\alpha \log N+\log d)^{2}+4(\log \beta)^{2}+3 d \beta^{2} N^{1-\alpha}
\end{aligned}
$$

and the second assertion follows.

Remark 2.2. Consider the SK model at the critical temperature $\beta_{c}$. Recall from Talagrand (2011b, Chapter 11) that there exists a constant $C>0$ such that $\mathbb{E}\left\langle R\left(\sigma^{1}, \sigma^{2}\right)^{2}\right\rangle \leq C / \sqrt{N}$ for all $N \geq 1$. Also, it is known (see Chatterjee, 2009) that $t \in[0,1] \mapsto \mathbb{E}\left\langle R(\sigma, \rho)^{2}\right\rangle_{t, 0}$ is a nondecreasing function with $\mathbb{E}\left\langle R(\sigma, \rho)^{2}\right\rangle_{1,0}=$ $\mathbb{E}\left\langle R\left(\sigma^{1}, \sigma^{2}\right)^{2}\right\rangle$. These imply that $\mathbb{E}\left\langle R(\sigma, \rho)^{2}\right\rangle_{t, 0} \leq C / \sqrt{N}$ for all $t \in[0,1]$. Consequently, from (2.2), $\operatorname{Var}\left(F_{N}\left(\beta_{c}\right)\right) \leq C \beta_{c}^{2} \sqrt{N}$.

Remark 2.3. Another interesting example to consider is the pure $p$-spin model with $p \geq 3$, i.e., $\mathbb{E} H_{N}\left(\sigma^{1}\right) H_{N}\left(\sigma^{2}\right)=N R\left(\sigma^{1}, \sigma^{2}\right)^{p}$. In this case, the determination of the critical temperature $\beta_{p, c}$ for the corresponding free energy was studied in Chen $(2019+)$ and it was established there that $\operatorname{Var}\left(F_{N}(\beta)\right) \asymp N^{1-p / 2}$ for all $\beta<\beta_{p, c}$. The current approach however does not seem to work at the criticality. 


\section{Acknowledgements}

Both authors thank Sourav Chatterjee for explaining Talagrand's upper bound for the variance of the free energy at the critical temperature and Erik Bates for the illuminating discussion and careful reading. They also thank the anonymous referee for providing many useful suggestions regarding the presentation of the paper. The first author's research is partially supported by NSF grants DMS-17-52184.

\section{References}

M. Aizenman, J. L. Lebowitz and D. Ruelle. Some rigorous results on the Sherrington-Kirkpatrick spin glass model. Comm. Math. Phys. 112 (1), 3-20 (1987). MR904135.

T. Aspelmeier. Free-energy fluctuations and chaos in the sherrington-kirkpatrick model. Phys. Rev. Lett. 100, 117205 (2008). DOI: 10.1103/PhysRevLett.100.117205.

T. Aspelmeier, A. Billoire, E. Marinari and M. A. Moore. Finite-size corrections in the Sherrington-Kirkpatrick model. J. Phys. A 41 (32), 324008, 21 (2008). MR2425779.

S. Chatterjee. Disorder chaos and multiple valleys in spin glasses. ArXiv Mathematics e-prints (2009). arXiv: 0907.3381.

S. Chatterjee. Superconcentration and related topics. Springer Monographs in Mathematics. Springer, Cham (2014). ISBN 978-3-319-03885-8; 978-3-319-03886-5. MR3157205.

S. Chatterjee. A general method for lower bounds on fluctuations of random variables $(2019+)$. To appear in Ann. Probab.

W-K. Chen. Phase transition in the spiked random tensor with rademacher prior $(2019+)$. To appear in Ann. Statist.

F. Guerra and F. L. Toninelli. Quadratic replica coupling in the SherringtonKirkpatrick mean field spin glass model. J. Math. Phys. 43 (7), 3704-3716 (2002). MR1908695.

M. Mézard, G. Parisi and M. A. Virasoro. Spin glass theory and beyond, volume 9 of World Scientific Lecture Notes in Physics. World Scientific Publishing Co., Inc., Teaneck, NJ (1987). ISBN 9971-50-115-5; 9971-50-116-3. MR1026102.

D. Panchenko. The Sherrington-Kirkpatrick model. Springer Monographs in Mathematics. Springer, New York (2013). ISBN 978-1-4614-6288-0; 978-1-4614-6289-7. MR3052333.

G. Parisi and T. Rizzo. Phase diagram and large deviations in the free energy of mean-field spin glasses. Phys. Rev. B 79, 134205 (2009). DOI: 10.1103/PhysRevB.79.134205.

D. Sherrington and S. Kirkpatrick. Solvable model of a spin-glass. Phys. Rev. Lett. 35, 1792-1796 (1975). DOI: 10.1103/PhysRevLett.35.1792.

M. Talagrand. Spin glasses: a challenge for mathematicians. Cavity and mean field models, volume 46. Springer-Verlag, Berlin (2003). ISBN 3-540-00356-8. MR1993891.

M. Talagrand. Mean field models for spin glasses. Volume I. Basic examples, volume 54. Springer-Verlag, Berlin (2011a). ISBN 978-3-642-15201-6. MR2731561. 
M. Talagrand. Mean field models for spin glasses. Volume II. Advanced replicasymmetry and low temperature, volume 55. Springer, Heidelberg (2011b). ISBN 978-3-642-22252-8; 978-3-642-22253-5. MR3024566. 